\title{
Author Index Vol. 59, 1997
}

Abe,S. 159 Akagi, H. 235 Albegger, K. 23 Albers, F.W.J. 135 Amedee, R.G. 209 Anniko, M. 258,311 Aoki,A. 91 Arens, C. 179 Arnold, W. 23, 79 Asai,M. 296 Asakura, K. 238, 286 Asian, A. 18

Backes,K. 317 Balyan,F.R. 18 Baumann, U. 23 Baumgartner, W. 23 Begall, K. 23,209

Bende,M. 36 Bernstein, J. 10 Bohndorf, M. 23 Bordure,P. 170 Bruijn, A.J.G. de 277

Caylan,R. 18 Cheng, K.-C. 10,215 Chetty,R. 336

Deli,R. 166 Denk,D.-M. 100 Donnellan,R. 336 Döring, H. 23 Dreyer, T. 179

Ecke,U. 209 Ehrenberger, K. 23 Eistert,B. 179 El-Degwy, A.A. 332 El-Sharabasy, A.E. 332

Endo,K. 269

Fini-Storchi, I. 182 Forgbert, O. 23 Freigang, B. 23 Friedlander, G. 170 Frosini,P. 182 Frusoni, F. 166 Fujiyoshi, T. 215 Fukaya,T. 296

Gammert, Ch. 23 Ganzer,U. 79

García-Berrocal, J.R. 64 Gerhardt, H.J. 23 Ghonim,M.R. 332 Glanz,H. 179 Goumas,P.D. 106 Govender, D. 336 Graf, P. 39 Granström, G. 147 Grudemo, H. 50,280 Gstöttner,W. 23 Hagen,R. 115 Hallén,H. 39 Harabuchi,Y. 238 Harada,Y. 4,263 Haraguchi, H. 60 Hasegawa, M. 60, 141 Häusler,R. 23 He,D.Z.Z. 243 Heinemann, M. 23 Heinrich, U.-R. 317 Helms, J. 23 Henley, CM. 73 Hentona, H. 272 Herman, P. 170 Hinriksdóttir, I. 36 Hochmair-Desoyer, 1.23 Hofmann, E. 115 Hofmann, G. 23 Hoppe, F. 115 Hsu,W.-J. 258 Huang, T.-S. 258 Hüttenbrink, K. 23

Igarashi,M. 269 Iguchi, H. 230 Iida,M. 269 Ikeda,K. 45 Ikeda,M. 326 Ilberg, C. von 23 Inoue, M. 292 Ishibashi,T. 296 Ishida,K. 269 Itaya,T. 155 Ito,J. 230 Ito,K. 122 Jacobsson, C. 147 Janssen, T. 23 Jenkins, H.A. 73 Jones, TJ. 339 Juto,J.-E. 50,280

Kaider,A. 100

Kanzaki,J. 159

Kärjä,V. 97

Kastenbauer, E. 23

Kataura,A. 238,286

Kato,A. 230

Kau,R.J. 79

Kiefer,J. 23

Kimura, R.S. 84

Kingma,H. 198

Kitajima, K. 155

Kitano,H. 155

Klemi,P. 119

Komatsuzaki, A. 60, 67, 193, 272

Korves, B. 23

Krug,M.S. 10,215

Kubo,T. 230,258 
Kumar, B.N. 176,339

Kunihiro,T. 159

Lin,X. 243 Lippert, K.L. 23 Little, J.T. 176 Littman, T.A. 73 Liu,T.-C. 131,243,322

Maeda,S. 60

Mai, J.K. 127

Mair,A. 23

Mann,W. 23,317

Mann,W.J. 209

Marianowski, R. 170

Mastorakou, A.N. 106

Mastronikolis, N.S. 106

Masuda,Y. 235

Masutani,H. 230

Mathis,A. 23

Maurer,J. 317

Maurizi, M. 166

Meyer zum Gottesberge, A.M. 127

Mizuno, M. 122

Mizutani,J. 122

Morita, I. 193

Moser, L. 23

Müller,J. 23

Müller-Aschoff, E. 23

Murofushi,T. 122

Murphy, C. 36

Nadol, J.B., Jr. Naito,H. 248 Naitoh,A. 269

KARGER

E-Mail karger@karger.ch Fax + 41613061234 http://www. karger. ch

(C)1997S. KargerAG, Basel

343

Nakagawa, T. 303 Nakai, Y. 230, 303 Nikiforidis, G.C. 106 Nishizaki, K. 235 Noguchi, Y. 272

Nomura, K. 269 Nomura, Y. 91 Norris,C.H. 209

Ogawa, T. 235 Ogura, M. 45 Ohashi,K. 60 Oku,T. 141 Okuno,H. 67 Onoda,K. 235 Oshima,T.

45 Ozturan, O. 73

Paludetti, G. 166 Pfennigdorf, T. 23 Pinilla,M. 64 Premachandra, DJ. 57

Ramirez-Camacho, R. 64 Ramsden, R. 23 Ribari, O. 23 Rosingh,H.J. 135 Rughubar, K. 336

Russo, A. 18

Sakai,M. 269 Sakashita,T. 230 Sakikawa,Y. 84 Sanna,M. 18 Satoh,Y. 159 Scarano, E. 166

Schilling, V. 23 Schlatter,T. 23 Schlöndorff, G. 23 Schön, F. 23 Schorn, K. 23 Sendtner-Gress, K. 79 Shibata,S. 303 Shido,F. 238 Shimomura, A. 45 Shintani, T. 286 Shiobara,R. 159

Shirasaki, H. 238 Sipilä,J. 119 Skarzynski, H. 23 Skinner, D.W. 339 Srinivasan, V. 57 Stephan, K. 23 Streitberger, Ch. 23 Suiter, A.M. 135 Sunami,K. 303 Suzaki,H. 91 Suzuki, H. 45 Suzuki, M. 10 Suzuki, N. 292 Syrjänen, K. 97 Syrjänen, S. 97

Taibah,A. 18 Takasaka, T. 45 Takayama, M. 230, 303 Takiguchi, T. 292 Takumida, M. 4,187,263,311 Tan,C.-T. 170 Tanaka,H. 67,272 Tange,R.A. 277 Tanno,N. 45 
Tatsuoka,H. 193 Terada, S. 292 Thumfart,W. 23 Titiz,A. 18 TranBaHuy,P. 170 Tsunoda, R. 296

Uchide,K. 292

Van Den Abbeele, T. 170 Vassilakos, P.J. 106 Vischer,M. 23

Wagner, H. 23 Wakashima, J. 238 Walsh, R.M. 176 Walter, N.M. 176 Watanabe,I. 326

Watanabe,K. 248 Wit,H.P. 135

Yajin,K. 4,263 Yamada, M. 60 Yamamoto, E. 155 Yamane, H. 230, 303 Yazawa, Y. 10,155

Yen,P.-T. 170 Yoo,T.-J. 10,215 Yoshihara,S. 159 Yoshimoto, S.-i. 296 Yuen,K. 235

Zenner,H.P. 23

Zhang, D.M. 4, 187,263,311

Ziese, M. 23

Zorowka, P. 23

344

ORLVol. 59, 1997

Author Index 